Check for updates

Cite this: J. Mater. Chem. C, 2020, 8, 15868

DOI: $10.1039 /$ d0tc90198a

rsc.li/materials-c

\section{Correction: Printed, cost-effective and stable poly(3-hexylthiophene) electrolyte-gated field-effect transistors}

\author{
Davide Blasi, ${ }^{a}$ Fabrizio Viola, ${ }^{\mathrm{b}}$ Francesco Modena, ${ }^{\text {bc }}$ Axel Luukkonen, ${ }^{\mathrm{d}}$ \\ Eleonora Macchia, ${ }^{\text {ad }}$ Rosaria Anna Picca, ${ }^{\text {ae }}$ Zahra Gounani, ${ }^{d}$ Amit Tewari, ${ }^{d}$ \\ Ronald Österbacka, ${ }^{\mathrm{d}}$ Mario Caironi, ${ }^{\mathrm{b}}$ Zsolt M. Kovacs Vajna, ${ }^{\mathrm{f}}$ Gaetano Scamarcio, ${ }^{\mathrm{g}}$ \\ Fabrizio Torricelli* ${ }^{f}$ and Luisa Torsi*ade
}

Correction for 'Printed, cost-effective and stable poly(3-hexylthiophene) electrolyte-gated field-effect transistors' by Davide Blasi et al., J. Mater. Chem. C, 2020, DOI: 10.1039/d0tc03342a.

The authors regret the omission of some details from the Acknowledgements section of the published article. The corrected Acknowledgements section is given below (the bold text indicates the amendments):

This work was partially carried out at PoliFab, the micro and nano-technology center of the Politecnico di Milano. PON SISTEMA (MIUR), H2020 - Electronic Smart Systems - SiMBiT: Single molecule bio-electronic smart system array for clinical testing (Grant agreement ID: 824946), Academy of Finland projects \#316881 and \#316883 "Spatiotemporal control of Cell Functions", Future in Research APQ Ricerca Regione Puglia "Programma regionale a sostegno della specializzazione intelligente e della sostenibilità sociale ed ambientale - FutureInResearch" - "BEND" Biosensori elettronici intelligenti per la diagnosi precoce di malattie neurodegenerative (B164PG8), "PMGB - Sviluppo di piattaforme meccatroniche, genomiche e bioinformatiche per l'oncologia di precisione" - ARS01_01195 - PON “RICERCA E INNOVAZIONE” 2014-2020 projects, the European Union, Italian Government and Lombardia Region for the project BIOSCREEN (POR FESR 2014-2020, ID number 1831459, CUP E81B20000320007), Åbo Akademi University CoE and CSGI are acknowledged for partial financial support.

The Royal Society of Chemistry apologises for these errors and any consequent inconvenience to authors and readers.

\footnotetext{
${ }^{a}$ CSGI (Centre for Colloid and Surface Science), 70125 Bari, Italy. E-mail: luisa.torsi@uniba.it

${ }^{b}$ Center for Nano Science and Technology@PoliMi, Istituto Italiano di Tecnologia, via G. Pascoli 70/3, 20133 Milan, Italy

${ }^{c}$ Dipartimento di Elettronica, Infomazione e Bioingegneria, Politecnico di Milano - Milano, Italy

${ }^{d}$ Faculty of Science and Engineering, Åbo Akademi University, 20500 Turku, Finland

${ }^{e}$ Dipartimento di Chimica, Università degli Studi di Bari "Aldo Moro", 70125 Bari, Italy

${ }^{f}$ Dipartimento Ingegneria dell'Informazione, Università degli Studi di Brescia, via Branze 38, 25123 Brescia, Italy. E-mail: fabrizio.torricelli@unibs.it

${ }^{g}$ Dipartimento Interateneo di Fisica “M. Merlin”, Università degli Studi di Bari “Aldo Moro”, 70125 Bari, Italy
} 